\title{
Topological asymmetry in the damping-pairing contribution of electron-boson scattering
}

\author{
G. Varelogiannis \\ Institute of Electronic Structure and Laser \\ Foundation for Research and Technology - Hellas \\ P.O. Box 1527, Heraklion, Crete 71110, Greece \\ and \\ M. Peter \\ DPMC, Université de Genève \\ 24 quai E. Ansermet, CH-1211 Genève 4, Switzerland
}

\begin{abstract}
We make a harmonic analysis of the pairing and damping contribution of a finite $k$ range isotropic electron-phonon (or other boson) scattering in an anisotropic two-dimensional electronic system. We show that the pairing contribution of the anisotropic part of the electronic system can be much larger than its damping contribution enhancing significantly $T_{c}$. The higher is the order of the harmonic of the electronic anisotropy, the higher can be the asymmetry in its damping-pairing contribution. This could explain the puzzle of a much broader quasiparticle peak in the n-doped than in the p-doped cuprates, their smaller $T_{c}$ 's being also attributed to larger damping effects.
\end{abstract}

PACS numbers: 74.25.Jb 74.20.-z 
The two-dimensional character of the electronic properties of cuprates is reflected in the structure of their Fermi surface. Measurements by Angular Resolved Photoemission Spectroscopy (ARPES) [1, 2] and Positron anhilation [3] indicate that the Fermi surface corresponding to the $\mathrm{CuO}$ planes is almost cylindrical. This is also the result of band structure calculations [4]. On the other hand some phase sensitive experiments in high$T_{c}$ superconductors report the possibility of the variation of sign of the order parameter in the $a b$ plane [5]. There is intense theoretical activity on the study of the high- $T_{c}$ phenomenology in terms of $\mathrm{d}$-wave pairing that could originate for example from the interaction of electrons with antiferromagnetic spin fluctuations [6].

An alternative interpretation of the anisotropies in terms of finite range in $k$ space electron-phonon scattering has been proposed recently by several groups in different contexts [7, 8, 9, 10, 11, 12, 13]. Such momentum modulation of the electron-phonon scattering could result from the strong Coulomb correlations of the carriers [14] reflecting the possibility of a phase separation instability [15] in Hubbard type models, or could be simply due to the two dimensional character of the electronic system [16]. We will consider here an analytically solvable model for an isotropic finite range (in k-space) electron-phonon scattering in a two dimensional electronic system which respects tetragonal symmetry. Our analysis is not specifically linked to phonons and is valid if phonons are replaced by other bosons provided the requirements of adiabaticity are fulfiled. Making an harmonic analysis of this model, we obtain an unexpected interference between electronic anisotropies and superconducting and damping properties. We show that the anisotropic part of the electronic system can contribute much more to the pairing than to the damping effects and this of course enhances significantly $T_{c}$. We also obtain a simple understanding of characteristic differences between the n-doped and p-doped ma- 
terials. Our analysis points out that within conventional theory of superconductivity, sharp variations of the electronic density of states can strongly favor high-T.

We consider a two-dimensional electronic system respecting tetragonal symmetry in the $a b$ plane. We also consider that the characteristic momenta exchanged during the pairing interaction are small compared to the characteristic momenta of the variations of the electronic Density of States (DOS) over the Brillouin zone. In that case we have different couplings in different regions of the Fermi surface, which are proportional to the local DOS [11]. Anisotropies in superconductivity reflect the DOS anisotropies since the electron-phonon matrix element is supposed for the moment isotropic. The momentum dependent Eliashberg function [17] can be written in the form of Fourier series respecting tetragonal symmetry [18]

$$
\alpha^{2} F \approx \sum_{M=0}^{\infty} F_{4 M}(\Omega) \cos \left(4 M \phi^{\prime}\right) \exp \left\{-A\left|\phi-\phi^{\prime}\right|\right\}
$$

The characteristic range of the interaction is $\phi_{0}=1 / A$. This type of Eliashberg function corresponds to the models considered in References [8, 11, 12, 13], details being in fact irrelevant. We consider here an exponential sharp cut-off for the momentum range of the interaction in order to obtain analytic results, however the form of the cut-off is not expected to have relevant implications as it has already been checked numerically in Ref. [12]. The $M=0$ term represents the homogeneous or isotropic part of the DOS. This term is dominant when the van Hove singularity is far from the Fermi level and the DOS is rather isotropic. When for example by doping the van Hove singularity is pushed close to the Fermi level at an energy distance of the order $\Omega$, the anisotropic terms of the interaction $(M \neq 0)$ become relevant.

In an analogous way the superconducting gap respects tetragonal symmetry and can also be written as a sum of Fourier series $\Delta=\sum_{M^{\prime}=0}^{\infty} \Delta_{\bar{M}} \cos (\bar{M} \phi)$ where $\bar{M}=4 M^{\prime}$ in the 
case of anisotropic s-wave gap or $\bar{M}=4 M^{\prime}+2$ in the case of an anisotropic d-wave gap. Both types of gap are accessible with an electron phonon coupling as that considered in eq. (1) depending in fact on the exact value and momentum structure of the Coulomb pseudopotential $\mu^{*}$. It is for example possible to have transitions between the two types of gap by adjusting the doping since $\mu^{*}$ is very sensitive on it [12]. With these notations it is not difficult to see that the renormalization due to the electron-phonon interaction becomes proportional to the integral

$$
I_{Z}(\phi)=\int_{0}^{2 \pi} d \phi^{\prime} \frac{\sum_{M=0}^{\infty} F_{4 M} \cos \left(4 M\left(\phi-\phi^{\prime}\right)\right) \exp \left\{-A\left|\phi-\phi^{\prime}\right|\right\}}{\sqrt{\omega_{m}^{2}+\left[\sum_{M^{\prime}=0}^{\infty} \Delta_{\bar{M}} \cos \left(\bar{M} \phi^{\prime}\right)\right]^{2}}}
$$

There is a non trivial relationship between the effective anisotropies of the interaction, and its pairing and damping contributions that has never been exploited up to now. We consider first the damping effects in the normal state. The general belief is that the larger is the interaction, larger are the damping effects no matter the topology of the electronic system. Here we will study for the first time the interference of the topology of the electronic system with the damping effects. In the normal state, the damping integral of equation (2) gives the following simple result

$$
I_{Z}(\phi)=\sum_{M=0}^{\infty} F_{4 M} \frac{2 A}{A^{2}+(4 M)^{2}} \cos (4 M \phi)
$$

The coefficients $F_{4 M}$ are specific parameters of a given electronic system, while the intrinsic properties of the anisotropic part of the interaction are contained in the ratio $2 A /\left(A^{2}+\right.$ $\left.(4 M)^{2}\right)$. We can make a very important remark here. The lower is the order of the DOS harmonic (the lower is $M$ ) the larger is the amplitude of its contribution to the damping because of the presence of $(4 M)^{2}$ in the denominator. This effect is more pronounced when $A$ is smaller (when the range of the interaction is larger). Therefore the damping contribution of the electron-phonon scattering is strongly dependent on the topology of 
the electronic system. High DOS anisotropy harmonics are irrelevant for the normal state damping effects or in other terms high DOS harmonics can couple with any boson field (including phonons) without affecting the effective mass of the carriers !

If for example by doping we brink the van Hove singularity closer to the Fermi surface enhancing $F_{4 M \neq 0}$, then contrary to the general belief we do not enhance in a significant way the damping effects in the normal state because of this destructive interference of the anisotropies of the electronic system with damping. We show in figure 1 the contribution to the damping effects of the $M=0, M=1$ and $M=2$ harmonics as a function of the characteristic range of the interaction $\phi_{0}$. Our approach is relevant to the order $1-\sin (\phi) / \phi$ and is therefore reasonable for angles up to at least $20^{\circ}$. We can see that for $\phi_{0}>5^{\circ}$, the larger $M$ harmonics begin to give significantly smaller damping contributions.

Having interactions that do not contribute to the damping is a very positive situation for superconductivity provided that these interactions contribute to the pairing. In fact, taking into account in a first approximation strong coupling effects, we can write $T_{c} \propto$ $\exp \left\{\left(1+\lambda_{Z}\right) / \lambda_{\Delta}\right\}$ where $\lambda_{Z}$ is the damping contribution of the Eliashberg function and $\lambda_{\Delta}$ its pairing contribution. In isotropic superconductors we have $\lambda_{\Delta} \approx \lambda_{Z}$. If now the high harmonics do not contribute to the damping but give a significant contribution to the pairing we may obtain $\lambda_{Z} \ll \lambda_{\Delta}$ which is the optimal condition for high- $T_{c}$.

Near $T_{c}$ the pairing contribution $\lambda_{\Delta}$ can be shown to be proportional to the integral

$$
\begin{aligned}
& I_{\Delta}(\phi)=\int_{0}^{2 \pi} d \phi^{\prime}\left[\sum_{M^{\prime}=0}^{\infty} \Delta_{\bar{M}} \cos \left(\bar{M} \phi^{\prime}\right)\right]\left[\sum_{M=0}^{\infty} F_{4 M}(\Omega) \cos \left(4 M \phi^{\prime}\right) \exp \left\{-A\left|\phi-\phi^{\prime}\right|\right\}\right]= \\
= & \sum_{M, M^{\prime}=0}^{\infty} F_{4 M} \Delta_{\bar{M}}\left[\frac{A}{A^{2}+(4 M+\bar{M})^{2}} \cos [(\bar{M}+4 M) \phi]+\frac{A}{A^{2}+(4 M-\bar{M})^{2}} \cos [(\bar{M}-4 M) \phi]\right]
\end{aligned}
$$


It is interesting to consider first the isotropic part of the DOS $(M=0)$ that reads

$$
I_{\Delta} \approx \frac{2 A}{A^{2}+\bar{M}^{2}} \cos (\bar{M} \phi)
$$

which is perfectly symmetric to the damping contribution given in equation (3) except that the order of the pairing harmonic $4 M$ is replaced by the order of the gap harmonic $\bar{M}$. The contribution of the isotropic part of the interaction $(M=0)$ to an isotropic gap $(\bar{M}=0)$ is therefore equal to its contribution to the damping, and this is the conventional expectation in isotropic superconductivity. However the contribution of the isotropic part of the interaction $(M=0)$ to higher gap harmonics $(\bar{M} \neq 0)$ is smaller and this appears natural. Indeed we expect an isotropic gap to be favored in the case of an isotropic electronic system. We show in figure (2) the $\phi_{0}$ dependence of the contribution of the $M=0$ DOS harmonic to the different gap harmonics. The symmetry of equations (3) and (5) gives a qualitative understanding of the electronic topology dependence of the damping effects. In fact the small damping contribution of the higher DOS harmonics $(M \neq 0)$ is equivalent to the smaller contribution of the isotropic part of the DOS $(M=0)$ to an anisotropic gap $(\bar{M} \neq 0)$.

We now consider the anisotropic part of the interaction and we analyze equation (4). If we have an isotropic gap $(\bar{M}=0)$ the pairing contribution (eq. 4$)$ is the same with the damping contribution (eq. 3). The contribution of a higher DOS harmonic $(M \neq 0)$ to an isotropic gap $(\bar{M}=0)$ is equal to its contribution to the damping. Therefore, although the anisotropic part of the interaction contributes less to the damping, if the gap is isotropic it also contributes less to the pairing and therefore we do not expect an enhancement of $T_{c}$ in that case.

The situation is totally different when the gap is anisotropic and has higher order harmonics $(\bar{M} \neq 0)$. The dominant term in equation (4) is the second one an can be 
significant when in the denominator $4 M-\bar{M}=0$. Comparing equations (3) and (4) it is easy to see that the contribution of a $M \neq 0$ DOS harmonic to the pairing is higher to its contribution to the damping when $4 M-\bar{M}=0$. We show in figure 3 the $\phi_{0}$ dependence of the pairing amplitude of the $M=1$ DOS harmonic for different gap harmonics: $\bar{M}=0$ (isotropic), $\bar{M}=2\left(d_{x^{2}-y^{2}}\right.$ gap) and $\bar{M}=4$ (anisotropic s component). The contribution to the $\bar{M}=0$ gap equals the damping contribution of this DOS harmonic. However the contribution of a given DOS $M \neq 0$ harmonic to the damping is not proportional to its contribution to $\bar{M} \neq 0$ pairing. In fact the contribution of the $M=1$ DOS harmonic to the d-wave $(\bar{M}=2)$ and anisotropic s-wave gap $(\bar{M}=4)$ is higher than its contribution to the damping (compare figs. 1 and 3). This of course enhances significantly the $T_{c}$ contribution of this DOS harmonic in the case of anisotropic gap. The effect is amplified when we consider higher order harmonics of the DOS anisotropies (higher values of M). We also remark that the higher is the DOS harmonic the smaller is the characteristic angle $\phi_{0}$ from which the pairing contribution becomes larger than the damping contribution of the interaction.

Of course all the previous contributions are weighted by the Fourier coefficients $F_{4 M}$ and $\Delta_{\bar{M}}$. The gap Fourier coefficients $\Delta_{\bar{M}}$ are obtained self consistently by the gap equation and therefore they also reflect the $F_{4 M}$ coefficients and the previously discussed pairing-damping contributions. The $F_{4 M}$ coefficients which describe the anisotropies of the electronic DOS are therefore the relevant material parameter for our discussion. To our approach the optimal situation for high- $T_{c}$ is the situation in which the weight of the higher order Fourier components $F_{4 M}$ is the larger. This can be obtained for example when the van hove singularity is close to the Fermi level and the electronic density of states variates sharply. 
Our arguments for the enhancement of $T_{c}$ are purely topological, and provide therefore a new perspective to the van Hove singularity approach to high- $T_{c}$. Up to now, the studies of the effect of the van Hove singularity in the self consistent Eliashberg framework have been done considering a totally isotropic system and reported the impossibility of this mechanism to produce a sharp enhancement of $T_{c}$ contrary to previous claims based on simple BCS [19]. In the light of our analysis, the effect on $T_{c}$ of the van Hove singularity will be amplified compared to that predicted in an isotropic situation, since the effective enhancement of the coupling (enhancement of the $F_{4 M \neq 0}$ ) acts more on the pairing than on the damping.

We can also obtain within our picture some insight on the essential differences between the n-doped and p-doped cuprates. The ARPES obtained quasiparticle peak near $E_{F}$ in the normal state of $\mathrm{Nd}_{2-x} C e_{x} C u O_{4-\delta}$ [20] (which is an n-type superconductor) is much broader than the corresponding peak in $\mathrm{YBa}_{2} \mathrm{Cu}_{3} \mathrm{O}_{7}$ and $\mathrm{BiSr}_{2} \mathrm{CaCu}_{2} \mathrm{O}_{8}$ [21]. In the case of $N C C O$ the van Hove singularity lies far below the Fermi level $($ at $\approx 300 \mathrm{meV}$ below $E_{F}$ ) and the anisotropic part of the DOS is very small. In that case the lowest order DOS harmonics are dominant and the whole electron-phonon coupling contributes to the damping and therefore the width of the quasiparticle peak (which reflects the damping effects) is rather large. In the case of the p-doped cuprates instead, the van Hove singularity is close to the Fermi level and we have significant high order harmonics in the DOS that contribute little to the width of the quasiparticle peak in the normal state since they are not effective for the damping. On the other hand the critical temperatures are much higher in the p-doped materials because the high DOS harmonics can have a significant contribution to an anisotropic s-wave or d-wave gap without contributing to the damping. This $T_{c}$ differences are present in our scheme even if the overall coupling 
between the two types of materials is of the same order.

Notice that within our analysis the anisotropic s-wave gap $\left(\bar{M}=4 M^{\prime}\right)$ is favored with respect to the anisotropic d-wave gap $\left(\bar{M}=4 M^{\prime}+2\right)$ because of the tetragonal DOS symmetry. This is also the result of numerical simulations when the Coulomb pseudopotential is neglected [11]. The situation can be inverted when the Coulomb pseudopotential reaches a critical value which depends on its characteristic momentum variations [12]. We understand however clearly that the anisotropic gap may result from anisotropic parts of the DOS (the isotropic DOS contributes very few to an anisotropic gap as it is shown in fig. 2) and therefore we can also understand why in the n-doped cuprates the gap seems isotropic while in the p-doped cuprates with the large $F_{4 M \neq 0}$ components the gap is very anisotropic and probably in some of them is of anisotropic d-wave type [12]. Notice finally that other situations that may lead to sharp variations in $k$-space of the electronic DOS on the Fermi level like the presence of CDW or nesting, could also be within our analysis favorable for high- $T_{c}$. 


\section{References}

[1] For a review on ARPES see: Z.-X. Shen and D.S. Dessau, Physics Reports 253, p. $1-162(1995)$

[2] J. Ma et al., Phys. Rev. B 51, 9271 (1995)

[3] M. Peter et al., Europhys. Lett. 18, 313 (1992)

[4] For a review see: W. Pickett, Rev. Mod. Phys. 61, 433 (1989)

[5] D.A. Wollman et al., Phys. Rev. Lett. 71, 2134 (1993); D.A. Brawner and H.R. Ott, Phys. Rev. B 50, 6530 (1994); C.C. Tsuei et al., Phys. Rev. Lett. 73, 593 (1994)

[6] N.E. Bickers, D.J. Scalapino and S.R. White, Phys. Rev. Lett. 62, 961 (1989); P. Monthoux, A. Balatsky and D. Pines, Phys. Rev. Lett. 67, 3348 (1991)

[7] S. Chakravarty et al., Science 261, 337 (1993)

[8] A.A. Abrikosov, Physica C 244, 243 (1995)

[9] G. Varelogiannis et al., Phys. Rev. B 52, R15753 (1995)

[10] J. Ruvalds et al., Phys. Rev. B 51, 3797 (1995)

[11] G. Varelogiannis et al., Phys. Rev. B 54, R6877 (1996)

[12] G. Varelogiannis, preprint cond-mat/9511139; G. Varelogiannis and M. Peter, Czech J. of Phys. 46, 1047 (1996)

[13] M. Weger, J. Low Temp. Phys. 95, 131 (1994); G. Santi, et al., J. of Supercond. 8, $215(1995)$ 
[14] M. Kulic and R. Zeyher, Phys. Rev. B 49, 4395 (1994)

[15] M. Marder, N. Papanicolaou and G.C. Psaltakis, Phys. Rev. B 40, 6920 (1990); A.N. Andriotis et al., Phys. Rev. B 47, 9208 (1993)

[16] H. Krakauer, W. Pickett and R.E. Cohen, Phys. Rev. B 47, 1002 (1993)

[17] J.P. Carbotte, Rev. Mod. Phys. 62, 1027 (1990)

[18] P.B. Allen, Phys. Rev. B 13, 1416 (1976)

[19] R.J. Radtke and M. Norman, Phys. Rev. B 50, 9554 (1994); R.J. Radtke, K. Levin, H.-B. Schüttler and M.R. Norman, Phys. Rev. B 48, 15957 (1993)

[20] D.M. King et al., Phys. Rev. Lett. 70, 3159 (1993); R.O. Anderson et al., Phys. Rev. Lett 70, 3163 (1993)

[21] T. Takahashi et al., Nature 334, 691 (1988); C.G. Olson et al., Science 245, 731 (1989); Y. Hwu et al., Phys. Rev. Lett., 67, 2573 (1991); J.G. Tobin et al., Phys. Rev. B 45, 5563 (1992); D. Dessau et al., Phys. Rev. Lett. 71, 2781 (1993) 


\section{Figure Captions}

Figure 1: The contribution to the damping of different DOS harmonics as a function of the characteristic range of the scattering $\phi_{0}$. Full line corresponds to $M=0$, the long-dashed line to $M=1$ and the short-dashed line to $M=2$

figure 2: The contribution to the pairing of the $M=0$ DOS harmonic as a function of $\phi_{0}$. Full line corresponds to $\bar{M}=0$, long dashed to $\bar{M}=2$ and short dashed to $\bar{M}=4$.

figure 3: Same as in figure 2 but for the $M=1$ DOS harmonic. 


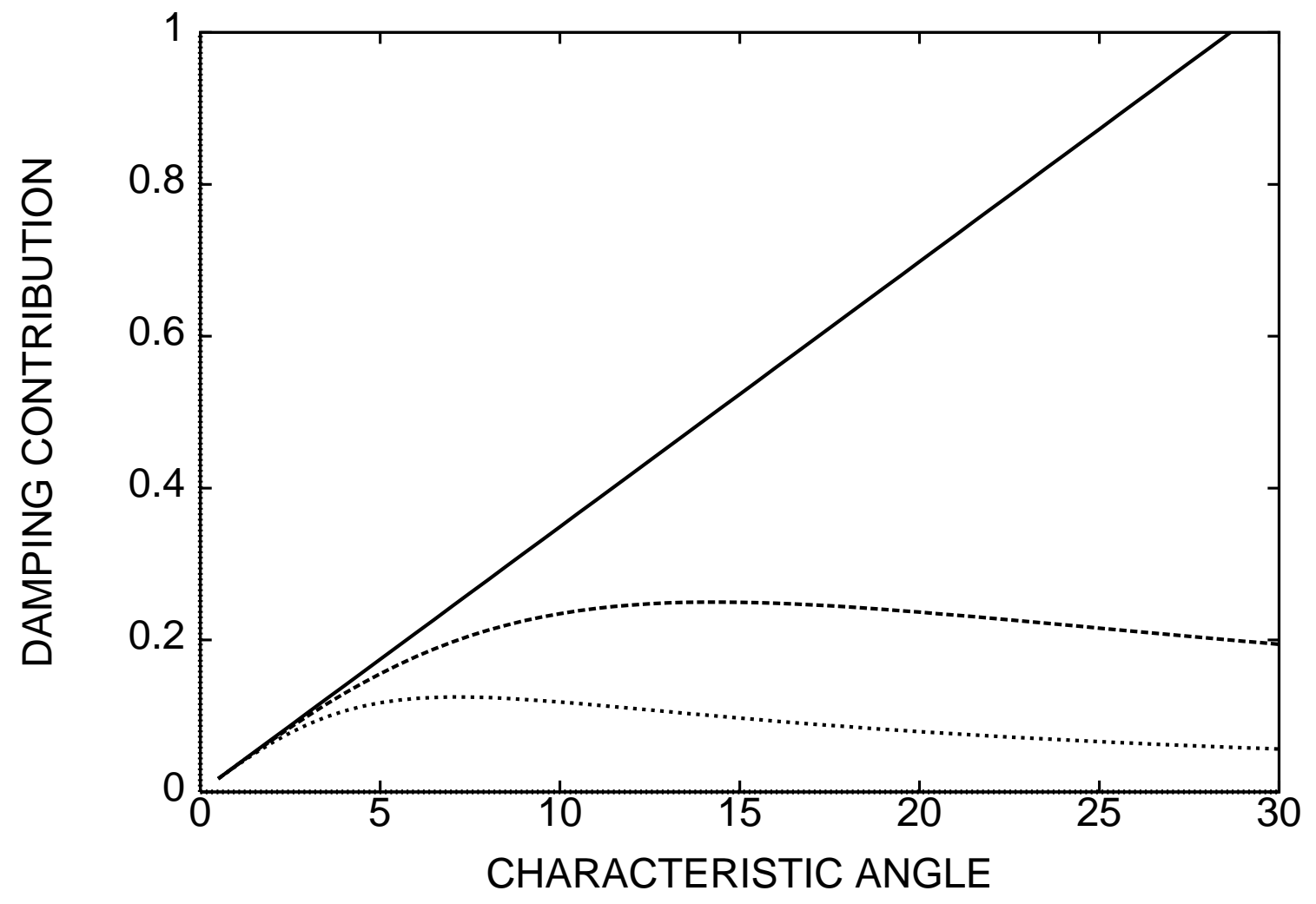




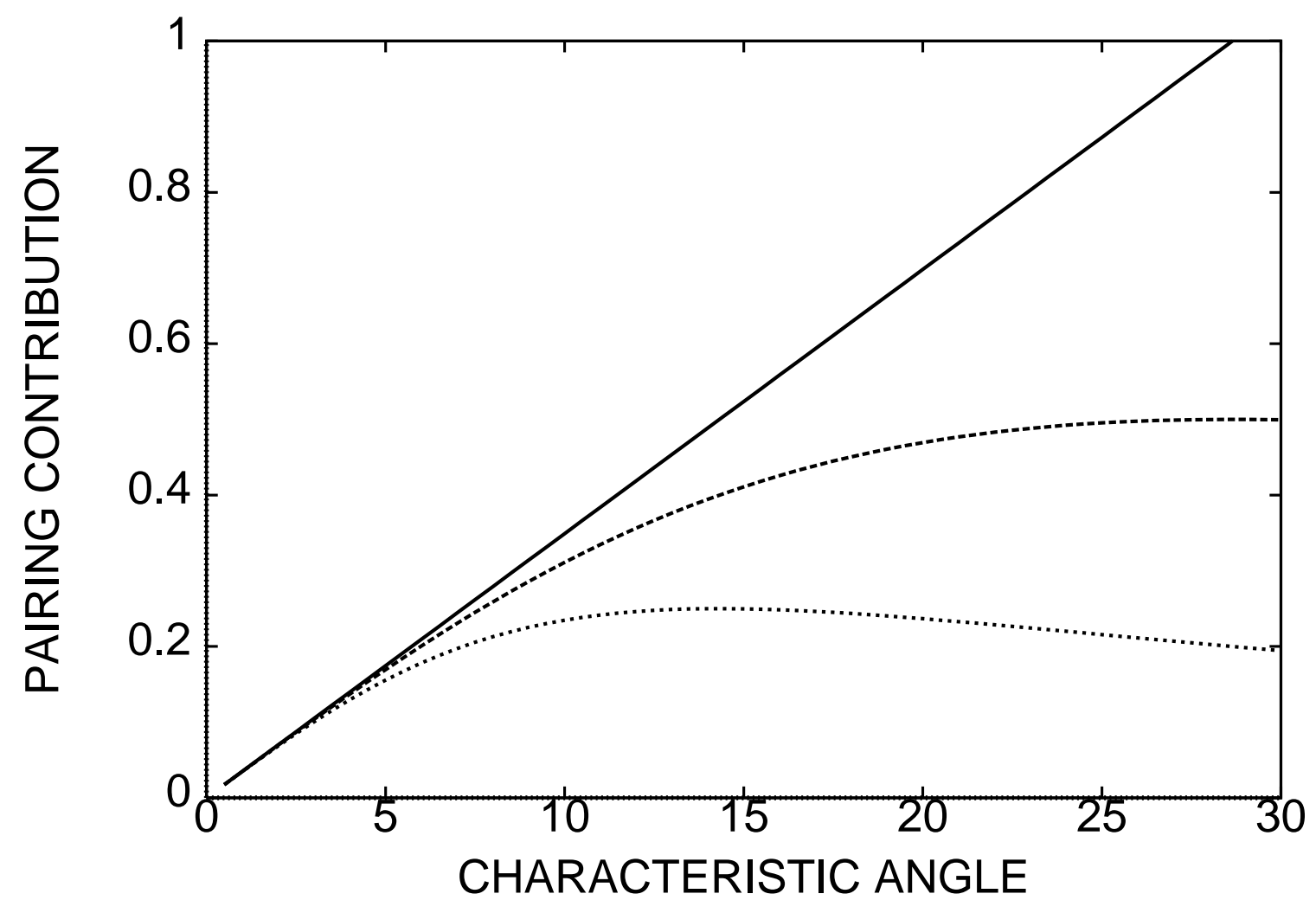




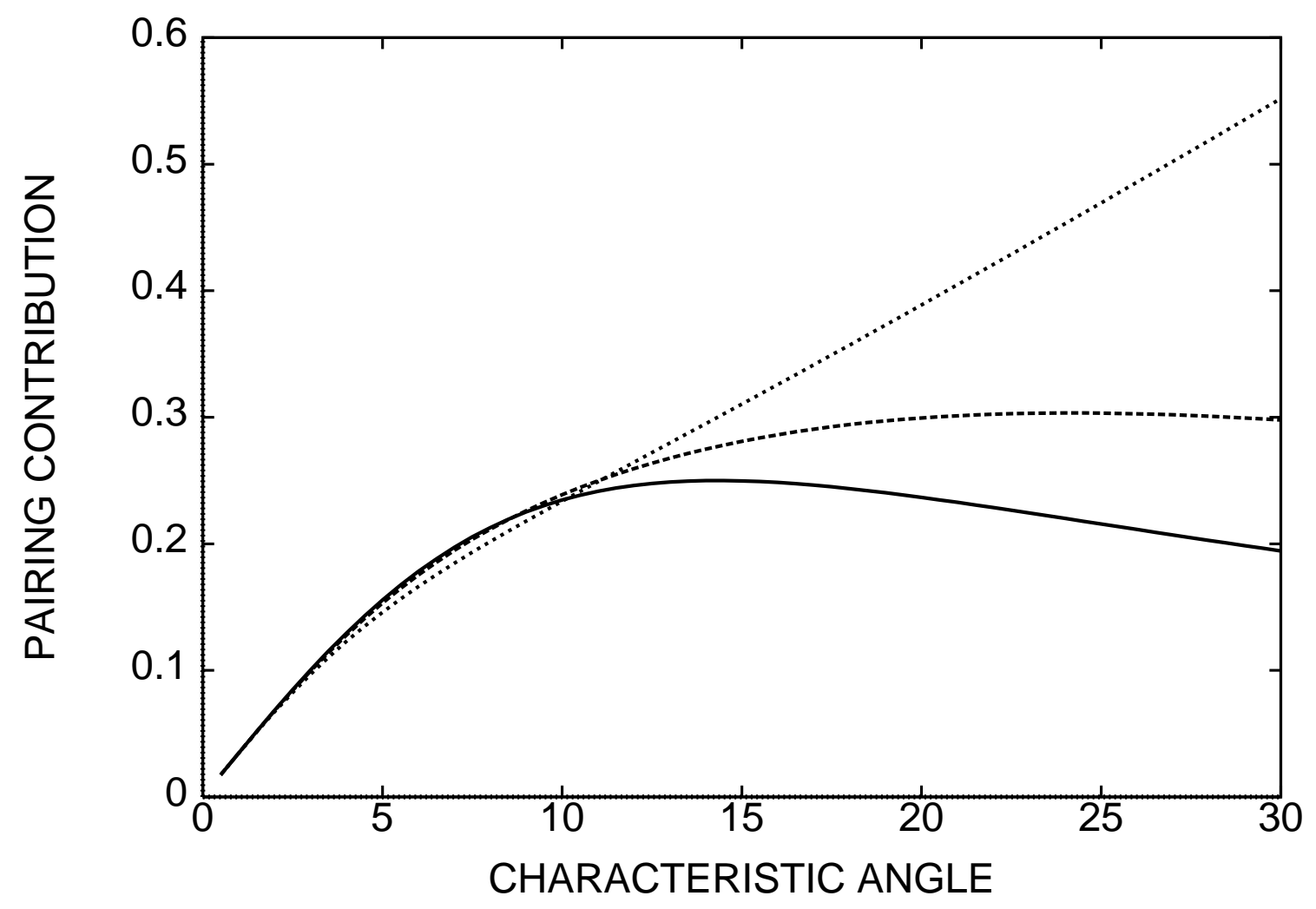

